\title{
ISOLATION OF LYTIC BACTERIOPHAGES INFECTING SALMONELLA TYPHIMURIUM AND SALMONELLA ENTERITIDIS
}

\author{
Zeliha Yildirim,* Tuba SAKIn and FATMa ÇOBAn \\ Niğde Ömer Halisdemir University, Faculty of Engineering, Department of Food Engineering, \\ Niğde, Turkey
}

(Received: March 21, 2018; accepted: April 24, 2018)

\begin{abstract}
The objectives of this study were to isolate, purify and determine host range of lytic bacteriophages infecting foodborne the pathogen Salmonella Typhimurium and S. Enteritidis. River/stream water, sewage, raw foods, wastewater from food processing plants, slaughterhouse and fish farms and water from troughs were used for the screening of bacteriophages. The richest sources in terms of phages infecting $S$. Typhimurium and Enteritidis were found to be sewage, wastewaters of slaughterhouse, food processing and fisheries and streams. A total of $33 \mathrm{~S}$. Typhimurium and $56 \mathrm{~S}$. Enteritidis phages were isolated and purified from the samples. It was demostrated that host ranges of the isolated phages were quite wide. The numbers of bacteria types inhibited by $S$. Typhimurium or Enteritidis phages were changed among 1-15 and $1-19$, respectively. It was found that $75.8 \%$ (25 out of 33 ) and $83.93 \%$ (47 out of 56) of isolated $S$. Typhimurium or Enteritidis phages formed clear plaques and were capable of lysing at least six or two Salmonella serovars. Beside Salmonella serovars, some $S$. Typhimurium (15 out of $33,45.5 \%)$ and $S$. Enteritidis phages (5 out of 56, 8.93\%) were also infective against $E$. coli strains. The host ranges of $S$. Typhimurium phages were wider than those of $S$. Enteritidis.
\end{abstract}

Keywords: Salmonella Typhimurium - Salmonella Enteritidis - bacteriophage - isolation - host range

\section{INTRODUCTION}

Salmonella enterica belong to the family Enterobacteriaceae and is an important zoonotic pathogen and is the primary cause of reported food poisoning worldwide. Non-typhoidal Salmonella enterica causes foodborne disease known as salmonellosis which is gastroenteritis foodborne illness in humans. Therefore, it is a major public health concern in many countries. Over 2500 serotypes of Salmonella are known, and the most common serovars in worldwide are $S$. Typhimurium and $S$. Enteritidis, causing salmonellosis $[7,12]$.

Bacteriophages or phages are natural killers of bacteria and they are abundant in the environment, with an estimated ratio of 10:1 with their bacterial hosts. Phages are self-replicating and self-limiting and their replication occurs naturally as long as their host cells are present and they infect only their specific host. Considerably, usage of

*Corresponding author; e-mail address: zeliha.yildirim@ohu.edu.tr 
phages as biopreservative and therapy agents has been known to be safe and nontoxic. It is considered that phages offer a great advantage over antibiotics since they target only the pathogens of interest $[9,10,12]$.

Recently, the development of antibiotic-resistant 'super-bugs' have highlighted the need for alternative strategies to combat infectious diseases. Pathogenic bacteria with wide-spectrum antibiotic resistance have become a considerable public health hazard. The emerging number of multi-drug and other antimicrobial biocides resistance of $S$. Typhimurium and $S$. Enteritidis are causing major concern among medical and veterinary health professionals and food industry $[24,28]$. An old strategy of using bacteriophages to challenge infections and prevent foodborne contamination and diseases is regaining popularity [27]. Therefore, many phage researches in the past two decades focused on phages infecting foodborne pathogenic bacteria such as Salmonella enterica, E. coli O157:H7, Campylobacter jejuni and Listeria monocytogenes $[17,20,21]$. Actually, a number of phage products which are used as biopreservative agents on ready-to-eat foods have been granted the "generally recognized as safe" (GRAS) status in the United States [19].

Phages have also been isolated capable of infecting $S$. Typhimurium and $S$. Enteritidis associated with foodborne illnesses. Most of $S$. Typhimurium and $S$. Enteritidis phages have narrow host ranges, which limits their use as biocontrol agents in food industry [7]. The aim of this work was to isolate $S$. Typhimurium and $S$. Enteritidis phages with broad infective spectrum to fight against these foodborne pathogenic bacteria in food industry and to have a collection of lytic bacteriophages against $S$. Typhimurium and $S$. Enteritidis.

\section{MATERIALS AND METHODS}

\section{Bacterial strains and culture conditions}

$S$. Typhimurium and $S$. Enteritidis strains used in this study were listed in Table 1 . The serovars were obtained from our culture collection, culture collection of Biology Department of Ankara University, Veterinary Faculty of Kırıkkale University and Food Engineering Department of Middle East University. The serovars were kept at $-80{ }^{\circ} \mathrm{C}$ in brain heart infusion broth $(5 \mathrm{~g} / \mathrm{L}$ beef heart (infusion from $250 \mathrm{~g}), 12.5 \mathrm{~g} / \mathrm{L}$ calf brains (infusion from $200 \mathrm{~g}$ ), $2.5 \mathrm{~g} / \mathrm{L}$ disodium hydrogen phosphate, $2 \mathrm{~g} / \mathrm{L} \mathrm{D}(+)$ glucose, $10 \mathrm{~g} / \mathrm{L}$ peptone, $5 \mathrm{~g} / \mathrm{L}$ sodium chloride, $\mathrm{pH}$ 7.4 \pm 0.2 ) (BHI, Merck, Darmstadt, Germany) with $20 \%$ glycerol.

\section{Collection and preparation of samples for bacteriophage screening}

The samples used in bacteriophage screening were randomly taken from river/stream water, sewage water, raw foods (milk, fruit, vegetable and meat), wastewater from food processing plants, fish farms and slaughterhouse, and water from troughs found 
Table 1

Bacteriophages infecting Salmonella Enteritidis and Salmonella Typhimurium

\begin{tabular}{|c|c|c|c|c|c|c|}
\hline Sample & Name of phage & $\begin{array}{l}\text { Host Serovar } \\
\text { Salmonella } \\
\text { Enteritidis }\end{array}$ & $\begin{array}{c}\text { Number of } \\
\text { phage (PFU/ml) }\end{array}$ & Name of phage & $\begin{array}{l}\text { Host Serovar } \\
\text { Salmonella } \\
\text { Typhimurium }\end{array}$ & $\begin{array}{c}\text { Number of } \\
\text { phage (PFU/ml) }\end{array}$ \\
\hline \multirow{9}{*}{$\begin{array}{l}\text { Rivers/ } \\
\text { streams }\end{array}$} & SE-Phage-1 & DMC22 & $152 \times 10^{8}$ & ST-Phage-1 & $\begin{array}{c}\text { Wild type } \\
14028\end{array}$ & $65 \times 10^{8}$ \\
\hline & SE-Phage-2 & DMC8 & $24 \times 10^{6}$ & ST-Phage-2 & AİBÜ & $42 \times 10^{4}$ \\
\hline & SE-Phage-3 & $\mathrm{DMC} 22$ & $50 \times 10^{7}$ & ST-Phage-3 & AİBÜ & $98 \times 10^{7}$ \\
\hline & SE-Phage-4 & DMC94 & $46 \times 10^{4}$ & ST-Phage-4 & LT2 SR II & $170 \times 10^{6}$ \\
\hline & SE-Phage-5 & MET-S1-411 & $32.5 \times 10^{6}$ & ST-Phage-5 & ATTC 14028 & $58 \times 10^{6}$ \\
\hline & SE-Phage-6 & MET-S1-742 & $30 \times 10^{7}$ & & & \\
\hline & SE-Phage-7 & MET-S1-411 & $142 \times 10^{9}$ & & & \\
\hline & SE-Phage-8 & ATCC 13075 & $110 \times 10^{7}$ & & & \\
\hline & SE-Phage-9 & DMC94 & $63 \times 10^{6}$ & & & \\
\hline \multirow{11}{*}{ Sewage } & SE-Phage-10 & DMC31 & $35 \times 10^{5}$ & ST-Phage-6 & LT2 SR II & $50 \times 10^{6}$ \\
\hline & SE-Phage-11 & M411 & $28 \times 10^{6}$ & ST-Phage-7 & AİBÜ & $27 \times 10^{6}$ \\
\hline & SE-Phage-12 & M742 & $82 \times 10^{6}$ & ST-Phage-8 & $\begin{array}{c}\text { Wild type } \\
14028\end{array}$ & $35 \times 10^{6}$ \\
\hline & SE-Phage-13 & ATCC 13075 & $43 \times 10^{6}$ & ST-Phage-9 & LT2 SR II & $102 \times 10^{8}$ \\
\hline & SE-Phage-14 & DMC8 & $63.5 \times 10^{5}$ & ST-Phage-10 & LT2 SR II & $37.5 \times 10^{7}$ \\
\hline & SE-Phage-15 & DMC22 & $56 \times 10^{6}$ & ST-Phage-11 & LT2 SR II & $90 \times 10^{7}$ \\
\hline & SE-Phage-16 & DMC3 & $42 \times 10^{9}$ & ST-Phage-12 & AİBÜ & $42 \times 10^{5}$ \\
\hline & SE-Phage-17 & DMC8 & $30,3 \times 10^{7}$ & ST-Phage-13 & ATTC 14028 & $137 \times 10^{4}$ \\
\hline & SE-Phage-18 & DMC8 & $66 \times 10^{4}$ & ST-Phage-14 & $\begin{array}{c}\text { Wild type } \\
14028\end{array}$ & $25.5 \times 10^{8}$ \\
\hline & SE-Phage-19 & ATCC 13075 & $135 \times 10^{9}$ & ST-Phage-15 & M625 & $64 \times 10^{5}$ \\
\hline & SE-Phage-20 & ATCC 13075 & $46 \times 10^{7}$ & & & \\
\hline \multirow{3}{*}{ Raw food } & SE-Phage-21 & DMC94 & $54 \times 10^{6}$ & ST-Phage-16 & $\operatorname{Tr} 90$ & $145 \times 10^{4}$ \\
\hline & SE-Phage-22 & DMC94 & $124 \times 10^{4}$ & ST-Phage-17 & Tr87 & $12 \times 10^{5}$ \\
\hline & SE-Phage-23 & DMC31 & $33 \times 10^{4}$ & & & \\
\hline \multirow{7}{*}{$\begin{array}{l}\text { Fisheries } \\
\text { wastewater }\end{array}$} & SE-Phage-24 & DMC22 & $64 \times 10^{7}$ & ST-Phage-18 & $\begin{array}{c}\text { Wild type } \\
14028\end{array}$ & $44 \times 10^{6}$ \\
\hline & SE-Phage-25 & KÜVF29 & $49 \times 10^{6}$ & ST-Phage-19 & AİBÜ & $38 \times 10^{9}$ \\
\hline & SE-Phage-26 & DMC22 & $47.5 \times 10^{7}$ & ST-Phage-20 & ATTC 14028 & $27 \times 10^{6}$ \\
\hline & SE-Phage-27 & DMC31 & $34 \times 10^{6}$ & & & \\
\hline & SE-Phage-28 & ATCC 13075 & $43.5 \times 10^{3}$ & & & \\
\hline & SE-Phage-29 & MET-S1-411 & $98 \times 10^{9}$ & & & \\
\hline & SE-Phage-30 & DMC 3 & $30 \times 10^{8}$ & & & \\
\hline
\end{tabular}


Table 1 (cont.)

\begin{tabular}{|c|c|c|c|c|c|c|}
\hline Sample & Name of phage & $\begin{array}{c}\text { Host Serovar } \\
\text { Salmonella } \\
\text { Enteritidis }\end{array}$ & $\begin{array}{c}\text { Number of } \\
\text { phage }(\mathrm{PFU} / \mathrm{ml})\end{array}$ & Name of phage & $\begin{array}{l}\text { Host Serovar } \\
\text { Salmonella } \\
\text { Typhimurium }\end{array}$ & $\begin{array}{c}\text { Number of } \\
\text { phage (PFU/ml) }\end{array}$ \\
\hline \multirow{10}{*}{$\begin{array}{l}\text { Food } \\
\text { processing } \\
\text { wastewaters }\end{array}$} & SE-Phage-31 & DMC 94 & $38 \times 10^{5}$ & ST-Phage-21 & $\begin{array}{c}\text { Wild type } \\
14028\end{array}$ & $24 \times 10^{7}$ \\
\hline & SE-Phage-32 & DMC31 & $38 \times 10^{5}$ & ST-Phage-22 & $\begin{array}{c}\text { Wild type } \\
14028\end{array}$ & $55 \times 10^{6}$ \\
\hline & SE-Phage-33 & DMC94 & $73 \times 10^{6}$ & ST-Phage-23 & $\begin{array}{c}\text { Wild type } \\
14028\end{array}$ & $21 \times 10^{5}$ \\
\hline & SE-Phage-34 & DMC 94 & $75 \times 10^{6}$ & ST-Phage-24 & ATTC 14028 & $64 \times 10^{4}$ \\
\hline & SE-Phage-35 & DMC8 & $82 \times 10^{6}$ & ST-Phage-25 & ATTC 14028 & $75 \times 10^{5}$ \\
\hline & SE-Phage-36 & MET-S1-742 & $41 \times 10^{9}$ & & & \\
\hline & SE-Phage-37 & DMC3 & $64 \times 10^{6}$ & & & \\
\hline & SE-Phage-38 & DMC22 & $98 \times 10^{7}$ & & & \\
\hline & SE-Phage-39 & DMC3 & $35 \times 10^{5}$ & & & \\
\hline & SE-Phage-40 & DMC22 & $45 \times 10^{7}$ & & & \\
\hline \multirow{12}{*}{$\begin{array}{l}\text { Slaughter- } \\
\text { house } \\
\text { wastewaters }\end{array}$} & SE-Phage-41 & DMC22 & $31 \times 10^{6}$ & ST-Phage-26 & $\operatorname{Tr} 87$ & $36 \times 10^{4}$ \\
\hline & SE-Phage-42 & DMC22 & $182 \times 10^{5}$ & ST-Phage-27 & $\begin{array}{c}\text { Wild type } \\
14028\end{array}$ & $42 \times 10^{5}$ \\
\hline & SE-Phage-43 & MET-S1-742 & $128 \times 10^{6}$ & ST-Phage-28 & AİBÜ & $63 \times 10^{6}$ \\
\hline & SE-Phage-44 & DMC3 & $50 \times 10^{5}$ & ST-Phage-29 & LT2 SR II & $28 \times 10^{6}$ \\
\hline & SE-Phage-45 & DMC31 & $102 \times 10^{2}$ & ST-Phage-30 & ATTC 14028 & $32 \times 10^{8}$ \\
\hline & SE-Phage-46 & KÜVF 29 & $20 \times 10^{6}$ & ST-Phage-31 & $\begin{array}{c}\text { Wild type } \\
14028\end{array}$ & $45.3 \times 10^{5}$ \\
\hline & SE-Phage-47 & MET-S1-411 & $123 \times 10^{6}$ & & & \\
\hline & SE-Phage-48 & DMC31 & $118 \times 10^{5}$ & & & \\
\hline & SE-Phage-49 & DMC22 & $15 \times 10^{9}$ & & & \\
\hline & SE-Phage-50 & MET-S1-742 & $21 \times 10^{4}$ & & & \\
\hline & SE-Phage-51 & ATCC 13075 & $59 \times 10^{5}$ & & & \\
\hline & SE-Phage-52 & MET-S1-742 & $46 \times 10^{5}$ & & & \\
\hline \multirow{4}{*}{ Troughs } & SE-Phage-53 & DMC8 & $65 \times 10^{8}$ & ST-Phage-32 & $\begin{array}{c}\text { Wild type } \\
14028\end{array}$ & $30.5 \times 10^{9}$ \\
\hline & SE-Phage-54 & DMC8 & $32 \times 10^{4}$ & ST-Phage-33 & AİBÜ & $104 \times 10^{5}$ \\
\hline & SE-Phage-55 & KÜVF 29 & $12 \times 10^{5}$ & & & \\
\hline & SE-Phage-56 & DMC31 & $37.5 \times 10^{7}$ & & & \\
\hline
\end{tabular}


in Niğde, Aksaray, Ankara and Kayseri provinces (Turkey). Liquid samples, except milk, were centrifuged at $6000 \times g$ for $15 \mathrm{~min}$ to remove solid particles and then the supernatants were passed through 0.45 and $0.22 \mu \mathrm{m}$ pore size sterile cellulose nitrate membrane filter (Sartorius, Germany). Milk samples were centrifuged at $6000 \times g$ for 15 min after addition of $10 \%$ lactic acid to precipitate casein and then the supernatants were filtered sterilized $(0.45 \mu \mathrm{m}$ pore size, cellulose nitrate). Semi-hard and solid samples were subjected to the following procedures: $25 \mathrm{~g}$ of the semi-solid and solid food samples were weighed in sterile conditions and placed in sterile stomacher bags and then $100 \mathrm{ml}$ of SM buffer (50 mM Tris-Cl, pH 7.5, $99 \mathrm{mM} \mathrm{NaCl}, 8 \mathrm{mM}$ $\mathrm{MgSO}_{4}, 0.01 \%$ gelatin (w/v)) were added. After homogenization in a stomacher for $2 \mathrm{~min}$, the samples were centrifuged and the filtrate was taken through a $0.45 \mu \mathrm{m}$ sterile cellulose nitrate membrane filter. All filtrate samples obtained from water and food samples were used for bacteriophages isolation. A total of 92 samples were analyzed.

\section{Isolation of bacteriophages}

Two methods were used for isolation of Salmonella bacteriophages: direct isolation and enrichment method. In the direct isolation protocol, filtered sample supernatants were directly used for bacteriophage screening against the test bacteria by the double agar layer plate method [1]. In the enrichment protocol, to increase the number of lytic phages, $20 \mathrm{ml}$ of the filtered samples were separately inoculated with $2 \mathrm{ml}$ of actively grown culture of 12 different $S$. Typhimurium strains ( $S$. Typhimurium LT2 SRII, MA1LT2/pNK972, MA53 T-POP, Tr90, Tr87, LT2 TH3923, SL 134, Wild type 14028, MET-S1-625, ATCC 14028, AIBU and AU) and $10 \mathrm{~S}$. Enteritidis strains (S. Enteritidis DMC3, DMC8, DMC22, DMC31, DMC94, ATCC 13075, KÜVF29, MET-S1-411, MET-S1-512, MET-S1-742) in BHI broth at $36 \pm 1{ }^{\circ} \mathrm{C}$ shaker and mixed with $3 \mathrm{ml}$ of double strength BHI broth. After incubation at $36 \pm 1{ }^{\circ} \mathrm{C}$ for 24 hours, chloroform $(50 \mu \mathrm{L} / \mathrm{mL})$ was added and vigorously mixed to ensure lysis of bacterial cells. The cultures were then centrifuged at $5000 \times \mathrm{g}$ for $15 \mathrm{~min}$ to remove cellular debris and supernatants were maintained at $5{ }^{\circ} \mathrm{C}$. Enriched samples were tested by double agar layer plaque assay against individual $S$. Typhimurium or $S$. Enteritidis strains [18].

\section{Double agar layer plaque assay}

Spot testing was used to determine the presence of anti-Salmonella bacteriophage. The host bacterial lawn was made by using soft BHI top agar ( $0.7 \%$ agar) containing host bacterial suspensions that were overlaid on top of BHI agar (1.5\% agar) plates. When the agar overlays were solidified, the samples were spotted onto the lawns and plates were incubated at $37^{\circ} \mathrm{C}$ for $24 \mathrm{~h}$. After incubation, all plates were examined for clear zone formation, resulted from the lysis of host bacterial cells. 
Phage enumerations were performed using the double-layer plaque titration method, using BHI as culture medium [1]. A $100 \mu \mathrm{L}$ of a dilution of the enriched or unenriched filtered phage samples and $300 \mu \mathrm{L}$ of the actively growing host bacterial cultures were added into BHI soft agar $\left(0.7 \%\right.$ agar) at $45-50{ }^{\circ} \mathrm{C}$ and after mixing well, soft BHI agar was poured onto (1.5\%) Petri dishes containing BHI agar (1.5\%). After solidification, plates were incubated at $37^{\circ} \mathrm{C}$ for $24 \mathrm{~h}$ and phage numbers were given as plaque forming units per milliliter $(\mathrm{PFU} / \mathrm{mL})$.

\section{Purification of bacteriophages}

For purification of the bacteriophages, a single plaque was picked using the large end of a sterile glass Pasteur pipette and the plaque was transferred to a sterile tube. The phages were diluted in SM buffer. After chloroform $(50 \mu \mathrm{l} / \mathrm{ml})$ extraction and centrifugation $\left(9,000 \times g, 20 \mathrm{~min}, 4^{\circ} \mathrm{C}\right)$, the supernatant was transferred to a new sterile tube. Serial dilutions made to obtain single phage plaques were inoculated into an early-log phase host culture, and the lysate was replated as described above. Bacteriophage purification process was repeated at least three times through plaque assay to make sure the removal of any contaminant phages. For determination of phage concentrations, tenfold serial dilutions of phage suspensions were prepared in SM buffer and then phage number were determine by using the double-layer method [26].

\section{Preparation of phages stocks}

The high titer phage stocks were prepared by inoculating $1 \mathrm{ml}$ of overnight host bacterial cultures with $100 \mu \mathrm{l}$ of purified phage stock into $100 \mathrm{ml}$ BHI broth and incubated overnight at $36 \pm 1{ }^{\circ} \mathrm{C}$ to allow amplification of the phage. After addition of chloroform $(50 \mu \mathrm{l} / \mathrm{ml})$ for complete lysis of the bacterial cells, the amplified phages were centrifuged at $8,000 \times \mathrm{g}$ for $15 \mathrm{~min}$ and the phage-rich supernatants were filtered through a disposable 0.45 or $0.22 \mu \mathrm{m}$ pore size syringe filter (cellulose acetate) (Sartorius, Germany) to eliminate bacterial contaminants. The filtrate was stored at either $4{ }^{\circ} \mathrm{C}$ until used or at $-80^{\circ} \mathrm{C}$ for long-term storage [15]. The titer of the phage stock was determined by the double-layer plaque titration method [1].

\section{Determination of host range of bacteriophages}

Besides $S$. Typhimurium and $S$. Enteritidis strains, $S$. Virchow DMC8, $S$. Infantis DMC7, $S$. Thompson DMC47, $S$. Anatum DMC90, $S$. Telaviv DMC62, $S$. Montavide DMC81, S. Kentucky DMC35, S. Carvalis DMC86, E. coli O157:H7 NCTC 12900, E. coli $\mathrm{O} 157: \mathrm{H} 7$ ATCC 43888, E. coli O157:H7 ATCC 35150, E. coli CFAI, E. coli ATCC 25922, Listeria monocytogenes ATCC 19115, Staphylococcus aureus ATCC 
25923, Bacillus cereus ATCC 10875, Yersinia enterocolitica O:9 AU, Citrobacter freundii AÜ, Enterobacter aerogenes AU and Enterococcus faecalis ATCC 29212 were used to investigate the inhibitory spectrum of Salmonella bacteriophages. Three hundred micro litter of exponential phase $\left(\mathrm{OD}_{600}=0.3\right)$ suspensions of the strain in BHI broth incubated at $36 \pm 1{ }^{\circ} \mathrm{C}$ in shaker $(100 \mathrm{rpm})$ were mixed in each BHI soft agar and then they poured onto the plates. After solidification of soft agar, $10 \mu \mathrm{l}$ of the $10^{-2}, 10^{-4}, 10^{-6}$ phage dilutions were spotted on the overlay and the plates were incubated at $36 \pm 1{ }^{\circ} \mathrm{C}$ for $24 \mathrm{~h}$. At the end of incubation, the plates were examined for plaques.

\section{RESULTS}

\section{Bacteriophage isolation}

In this study, a total of 92 samples were used for the screening of bacteriophages. Twelve out of 92 the samples were taken from river/stream water, 13 from sewage water, 10 from pool water of fish farms, 18 from raw foods (milk, fruit, vegetable and meat), 11 from wastewater of food processing plants, 13 from wastewater of slaughterhouse, and 8 from water of troughs. The data obtained as a result of phage screening were given in Table 1. As seen in Table 1, $S$. Typhimurium or $S$. Enteritidis bacteriophages found the most commonly in sewage, wastewaters of slaughterhouse, food processing and fisheries and streams. In the samples examined, the number of isolated bacteriophages infecting $S$. Typhimurium or $S$. Enteritidis was 33 and 56, respectively. Some of the isolated phages were given in Fig. 1.

Direct and enrichment method were used for isolation of bacteriophages. It was observed that the number of phages in the samples examined by the enrichment process increased considerably compared to the direct method. This increase was particularly pronounced in cases where the number of bacteriophages was low (e.g. food and river/stream waters).

\section{Phage purification and preparation of phage stocks}

Isolated phages were purified by using a single plaque method [1]. In purification process, a single plaque was taken from Petri dish containing maximum of 4-5 phage plaques and this process was repeated at least 3 times. All isolated phages were capable of lysing their host strains during the purification procedure. The purified bacteriophages were stored at $-80{ }^{\circ} \mathrm{C}$ in SM buffer containing $20 \%$ glycerol. It was determined that $S$. Typhimurium or $S$. Enteritidis bacteriophage numbers of the sample stocks were between $3.6 \times 10^{5}-3.8 \times 10^{10}$ and $1.02 \times 10^{4}-1.35 \times 10^{11} \mathrm{pfu} / \mathrm{ml}$, respectively. The environment in which 89 purified phages were isolated and their naming were summarized in Table 1. 


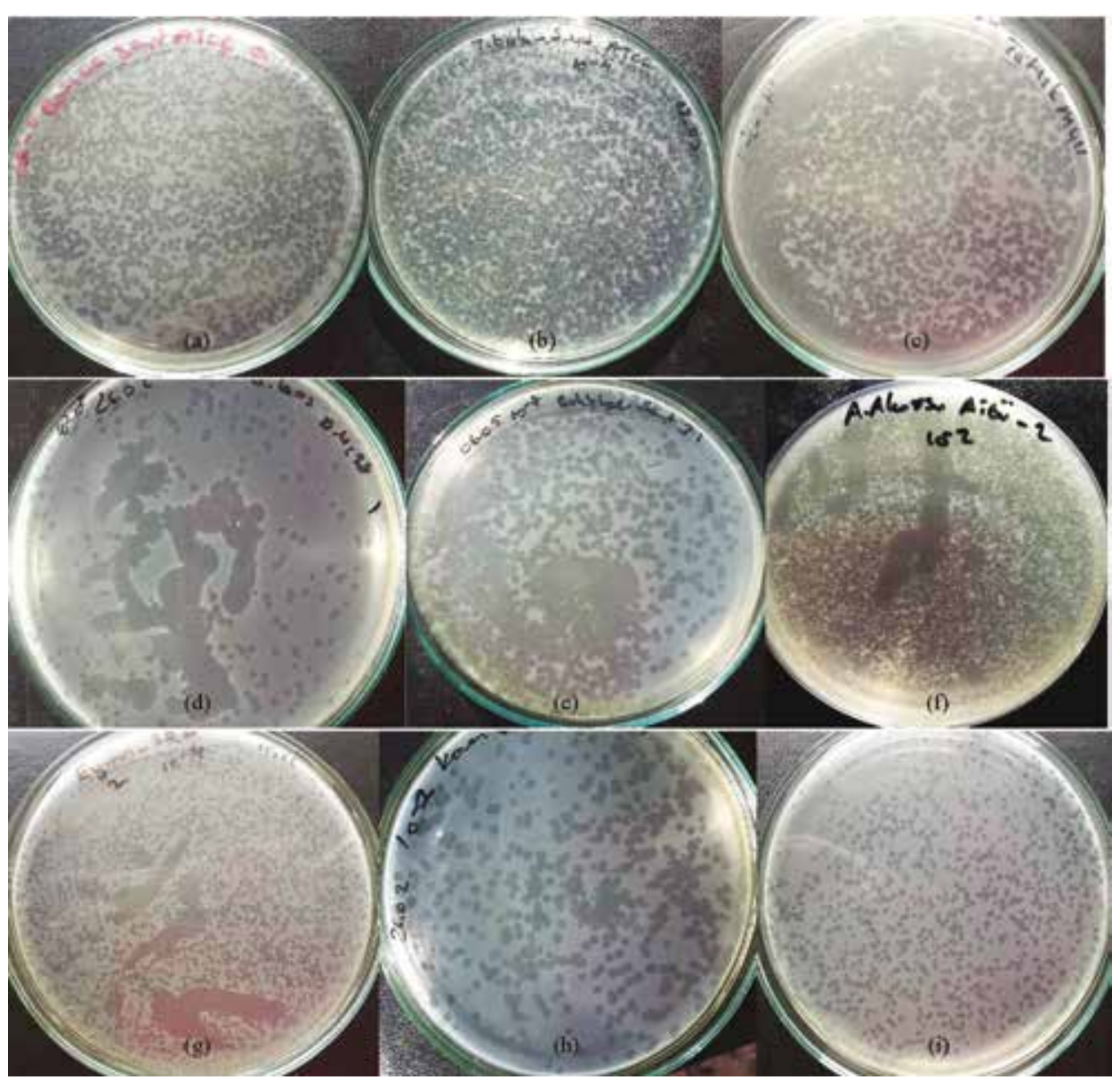

Fig. 1. Some isolated phages infecting Salmonella Enteritidis or Salmonella Typhimurium. (a) SE-Phage-17 from sewage, (b) SE-phage-19 from sewage, (c) SE-Phage-47 from slaughterhouse wastewaters, (d) SE-Phage-38 from food processing wastewater, (e) SE-Phage-48 from slaughterhouse wastewaters, (f) ST-Phage-3 from rivers, (g) ST-Phage-9 from sewage, (h) ST-Phage-24 from food processing wastewater, (i) ST-Phage-11 from sewage

\section{Host ranges of bacteriophages}

$S$. Typhimurium phages were able to lyse 3 to 18 of 36 strains tested and have highly changeable host ranges (Tables 2, 3). As seen in Tables 2 and 3, host ranges of most phages were wide except ST-Phage-16, 17 and 27 which were only infective against $S$. Typhimurium 2 to 4 serovars. Phages infective against $S$. Enteritidis also have wide host ranges, being to lyse 2 to 15 of 36 strains tested (Tables 3, 4). When ST-phages or SE-phages were screened by spot testing against a total of twenty-eight strains of Salmonella serovars including Typhimurium, Enteritidis, Virchow, Infantis, 
Table 2

Infective effect of Salmonella Typhimurium phages on Salmonella Typhimurium and Salmonella Enteritidis

\begin{tabular}{|c|c|c|c|c|c|c|c|c|}
\hline \multirow[b]{2}{*}{ Phages } & \multicolumn{8}{|c|}{ Salmonella Typhimurium } \\
\hline & SRII & MA1 & MA53 & $\operatorname{Tr} 90$ & $\operatorname{Tr} 87$ & TH3923 & SL134 & $\begin{array}{l}\text { Wild } \\
\text { type }\end{array}$ \\
\hline ST-Phage-1 & - & +++ & - & - & - & ++- & + & +++ \\
\hline ST-Phage-2 & ++- & ++- & - & - & - & ++- & + & +++ \\
\hline ST-Phage-3 & +++ & +++ & ++- & - & - & +++ & +++ & +++ \\
\hline ST-Phage-4 & +++ & + & + & - & + & ++- & ++- & + \\
\hline ST-Phage-5 & ++- & ++- & ++- & - & - & +++ & +++ & ++ \\
\hline ST-Phage-6 & +++ & ++- & ++- & - & + & +++ & ++- & + \\
\hline ST-Phage-7 & - & - & - & - & - & ++- & ++- & + \\
\hline ST-Phage-8 & +++ & ++- & ++- & - & - & ++- & ++- & ++- \\
\hline ST-Phage-9 & +++ & ++- & +++ & - & ++- & +++ & +++ & ++- \\
\hline ST-Phage-10 & +++ & +++ & +++ & - & ++- & ++- & +++ & ++- \\
\hline ST-Phage-11 & +++ & - & - & - & - & +++ & + & - \\
\hline ST-Phage-12 & - & - & - & - & - & + & + & - \\
\hline ST-Phage-13 & +- & - & +- & - & - & - & + & - \\
\hline ST-Phage-14 & - & +++ & - & - & - & +++ & +++ & +++ \\
\hline ST-Phage-15 & +++ & - & + & - & - & +- & - & +++ \\
\hline ST-Phage-16 & - & - & - & +++ & - & ++- & + & - \\
\hline ST-Phage-17 & - & - & - & ++- & +++ & + & - & - \\
\hline ST-Phage-18 & ++- & ++- & ++- & - & - & ++- & ++- & +++ \\
\hline ST-Phage-19 & - & +++ & - & - & - & +++ & ++- & +++ \\
\hline ST-Phage-20 & - & - & - & - & - & +++ & ++- & - \\
\hline ST-Phage-21 & +- & +++ & - & - & - & ++- & ++- & +++ \\
\hline ST-Phage-22 & - & ++- & - & - & - & +++ & +++ & ++ \\
\hline ST-Phage-23 & - & - & - & - & - & - & + & + \\
\hline ST-Phage-24 & - & - & - & - & - & ++- & +- & +++ \\
\hline ST-Phage-25 & - & - & + & - & +++ & - & + & - \\
\hline ST-Phage-26 & +++ & ++- & ++- & - & ++- & +- & ++- & - \\
\hline ST-Phage-27 & - & - & - & - & ++- & - & + & - \\
\hline ST-Phage-28 & +++ & ++- & ++- & - & - & +++ & +++ & ++- \\
\hline ST-Phage-29 & - & - & - & - & - & +++ & ++- & +++ \\
\hline ST-Phage-30 & - & +++ & - & - & + & +++ & - & +++ \\
\hline ST-Phage-31 & - & +++ & - & - & - & +++ & - & +++ \\
\hline ST-Phage-32 & - & - & - & - & - & +++ & +++ & +++ \\
\hline ST-Phage-33 & - & + & - & - & - & - & +- & +++ \\
\hline
\end{tabular}

$+++, 10^{-2}, 10^{-4}$ and $10^{-6}$ diluted phage samples were inhibitor positive; ++-, $10^{-2}, 10^{-4}$ diluted phage samples were inhibitor positive but $10^{-6}$ dilution sample was inhibitor negative;,$+- 10^{-2}$ dilution sample was inhibitor positive, $10^{-4}$ and $10^{-6}$ dilution sample was inhibitor negative; $-, 10^{-2}, 10^{-4}$ and $10^{-6}$ diluted phage samples were inhibitor negative 
Table 2 (cont.)

\begin{tabular}{|c|c|c|c|c|c|c|c|c|c|}
\hline \multicolumn{4}{|c|}{ Salmonella Typhimurium } & \multicolumn{6}{|c|}{ Salmonella Enteritidis } \\
\hline 625 & AïÜB & \begin{tabular}{|c|} 
MA1LT2/ \\
pNK972
\end{tabular} & $\begin{array}{c}\text { MA53 T- } \\
\text { POP }\end{array}$ & DMC8 & $\mathrm{DMC} 22$ & 13075 & 411 & 512 & 742 \\
\hline- & - & - & - & - & +++ & + & + & - & +++ \\
\hline++- & +++ & - & - & - & ++- & + & - & - & + \\
\hline+++ & +++ & - & - & +++ & +++ & +++ & +++ & - & +++ \\
\hline+ & + & - & - & + & ++- & +++ & +++ & - & +++ \\
\hline+++ & +++ & - & - & - & +++ & - & - & - & +++ \\
\hline+++ & + & - & - & +++ & +++ & +++ & + & - & + \\
\hline+++ & +++ & - & - & - & +++ & - & - & - & - \\
\hline+++ & +++ & - & - & +++ & +++ & +++ & +++ & - & +++ \\
\hline++- & + & - & - & ++- & - & +++ & + & - & - \\
\hline+++ & +- & - & - & ++- & +++ & +++ & +++ & - & +++ \\
\hline- & +- & - & - & - & +- & - & - & - & +- \\
\hline+ & - & - & - & - & + & - & - & - & + \\
\hline+ & +- & - & - & - & - & - & +++ & - & - \\
\hline- & - & - & - & +- & +++ & - & +- & - & +++ \\
\hline+++ & ++- & - & - & - & - & - & +++ & - & +++ \\
\hline- & - & - & - & - & - & - & - & - & - \\
\hline- & ++- & - & - & - & - & - & - & - & - \\
\hline- & - & - & - & - & +- & +- & +- & - & - \\
\hline- & +++ & - & - & - & +++ & - & - & - & +++ \\
\hline++- & - & - & - & - & +++ & - & - & - & ++- \\
\hline+- & - & - & - & - & +++ & +- & +- & - & +++ \\
\hline- & +++ & - & - & - & +++ & - & - & - & + \\
\hline- & - & - & - & - & - & - & - & - & - \\
\hline- & +++ & - & - & - & +++ & - & +++ & - & - \\
\hline+ & - & - & - & - & - & - & - & - & - \\
\hline+- & - & - & - & ++- & - & - & - & - & - \\
\hline+ & +++ & - & - & - & - & - & - & - & - \\
\hline+++ & ++- & - & - & - & +++ & - & - & - & +++ \\
\hline- & ++- & - & - & - & +++ & - & +++ & - & - \\
\hline- & ++- & - & - & - & +++ & - & +++ & - & - \\
\hline+ & ++- & - & - & - & +++ & - & +++ & - & - \\
\hline- & +++ & - & - & - & ++- & - & - & - & + \\
\hline+- & +++ & - & - & - & +++ & - & +++ & - & - \\
\hline
\end{tabular}


Table 3

Salmonella Typhimurium or Salmonella Enteriditis specific phages infecting other Salmonella serovar and Escherichia coli strains

\begin{tabular}{|c|c|c|c|c|c|}
\hline \multirow[b]{2}{*}{ Phages } & \multicolumn{5}{|c|}{ Other Salmonella enterica serovars } \\
\hline & $\begin{array}{l}\text { S. Virchow } \\
\text { DMC8 }\end{array}$ & S. Infantis DMC7 & $\begin{array}{l}S . \text { Thompson } \\
\text { DMC47 }\end{array}$ & $\begin{array}{l}\text { S. Anatum } \\
\text { DMC90 }\end{array}$ & $\begin{array}{l}S \text {. Telaviv } \\
\text { DMC62 }\end{array}$ \\
\hline \multicolumn{6}{|c|}{ ST-Phages } \\
\hline ST-Phage-1 & - & - & - & - & - \\
\hline ST-Phage-3 & - & - & - & +++ & - \\
\hline ST-Phage-4 & - & - & - & +++ & - \\
\hline ST-Phage-5 & - & - & - & - & - \\
\hline ST-Phage-6 & ++- & - & - & +++ & - \\
\hline ST-Phage-8 & - & - & +++ & +++ & - \\
\hline ST-Phage-9 & - & - & - & - & - \\
\hline ST-Phage-10 & - & - & - & +++ & - \\
\hline ST-Phage-12 & - & - & - & - & - \\
\hline ST-Phage-13 & - & - & - & - & - \\
\hline ST-Phage-15 & - & - & - & +++ & - \\
\hline ST-Phage-19 & - & - & - & ++- & - \\
\hline ST-Phage-20 & - & - & - & - & - \\
\hline ST-Phage-22 & - & - & ++- & +++ & - \\
\hline ST-Phage-23 & - & - & - & + & - \\
\hline ST-Phage-24 & - & - & - & - & - \\
\hline ST-Phage-25 & - & - & +++ & ++- & - \\
\hline ST-Phage-27 & - & - & - & - & - \\
\hline ST-Phage-28 & - & - & - & - & - \\
\hline ST-Phage-30 & - & - & - & - & - \\
\hline ST-Phage-32 & - & - & - & +++ & - \\
\hline ST-Phage-33 & - & - & - & - & - \\
\hline \multicolumn{6}{|c|}{ SE-Phages } \\
\hline SE-Phage-2 & - & - & - & - & - \\
\hline SE-Phage-3 & - & - & - & - & - \\
\hline SE-Phage-14 & - & - & - & - & - \\
\hline SE-Phage-16 & - & - & - & - & - \\
\hline SE-Phage-17 & - & - & - & - & - \\
\hline SE-Phage-24 & + & - & - & - & - \\
\hline SE-Phage-26 & - & - & - & - & - \\
\hline SE-Phage-30 & - & - & - & - & - \\
\hline SE-Phage-37 & - & - & - & - & - \\
\hline SE-Phage-39 & - & - & - & - & - \\
\hline
\end{tabular}

$+++, 10^{-2}, 10^{-4}$ and $10^{-6}$ diluted phage samples were inhibitor positive; $++-, 10^{-2}, 10^{-4}$ diluted phage samples were inhibitor positive but $10^{-6}$ dilution sample was inhibitor negative;,$+- 10^{-2}$ dilution sample was inhibitor positive, $10^{-4}$ and $10^{-6}$ dilution sample was inhibitor negative; $-, 10^{-2}, 10^{-4}$ and $10^{-6}$ diluted phage samples were inhibitor negative 
Table 3 (cont.)

Salmonella Typhimurium or Salmonella Enteriditis specific phages infecting other Salmonella serovar and Escherichia coli strains

\begin{tabular}{|c|c|c|c|c|c|c|c|}
\hline \multicolumn{3}{|c|}{ Other Salmonella enterica serovars } & \multicolumn{3}{|c|}{ Escherichia coli $\mathrm{O} 157: \mathrm{H} 7$} & \multicolumn{2}{|c|}{ E. coli } \\
\hline $\begin{array}{l}S . \text { Montavide } \\
\text { DMC } 81\end{array}$ & $\begin{array}{l}\text { S. Kentucky } \\
\text { DMC35 }\end{array}$ & $\begin{array}{l}\text { S. Carvalis } \\
\text { DMC86 }\end{array}$ & 12900 & 43888 & 35150 & CFAI & 25922 \\
\hline \multicolumn{8}{|c|}{ ST-Phages } \\
\hline- & - & - & - & + & - & - & - \\
\hline- & ++- & - & - & - & ++- & - & ++- \\
\hline- & ++- & - & - & - & - & - & - \\
\hline- & - & - & ++- & + & + & - & - \\
\hline- & ++- & - & ++- & - & - & - & - \\
\hline- & - & - & - & - & - & - & - \\
\hline- & - & - & +++ & +++ & +++ & - & - \\
\hline \multirow[t]{2}{*}{-} & ++- & - & +++ & - & - & - & - \\
\hline & - & - & + & - & - & - & - \\
\hline- & - & - & - & - & - & - & +++ \\
\hline- & +++ & - & - & - & - & - & - \\
\hline- & - & - & - & - & - & - & - \\
\hline- & - & - & +- & +- & +- & - & - \\
\hline- & - & - & - & + & - & - & - \\
\hline- & - & - & - & - & - & - & - \\
\hline- & - & - & +++ & +++ & +++ & - & - \\
\hline- & - & - & ++- & ++- & ++- & - & - \\
\hline- & - & - & ++- & +- & +- & - & - \\
\hline- & - & - & + & ++- & ++- & - & - \\
\hline- & - & - & +++ & +++ & +++ & - & - \\
\hline- & +++ & - & - & - & - & - & - \\
\hline- & +++ & - & - & - & - & - & +++ \\
\hline \multicolumn{8}{|c|}{ SE-Phages } \\
\hline- & - & - & - & - & - & - & ++- \\
\hline- & +++ & - & - & - & - & - & - \\
\hline- & +++ & - & - & - & - & - & - \\
\hline- & - & - & - & - & - & - & +++ \\
\hline- & - & - & - & + & - & - & - \\
\hline- & - & - & - & - & - & - & - \\
\hline- & +++ & - & - & - & - & - & - \\
\hline- & +++ & - & - & - & - & - & +++ \\
\hline- & - & +++ & - & - & - & - & - \\
\hline- & - & +++ & - & - & - & - & - \\
\hline
\end{tabular}


Table 4

Infective effect of Salmonella Enteritidis phages on Salmonella Enteritidis and Salmonella Typhimurium

\begin{tabular}{|c|c|c|c|c|c|c|c|}
\hline \multirow{2}{*}{ Phage } & \multicolumn{7}{|c|}{ Salmonella Enteritidis } \\
\hline & $\mathrm{DMC} 3$ & DMC8 & DMC22 & DMC31 & DMC94 & 13075 & 29 \\
\hline SE-Phage-1 & + & +++ & +++ & +++ & +++ & +++ & +++ \\
\hline SE-Phage-2 & + & +++ & +++ & + & ++- & + & - \\
\hline SE-Phage-3 & + & + & +++ & +++ & +++ & ++- & +++ \\
\hline SE-Phage-4 & - & - & +++ & - & +++ & +++ & +++ \\
\hline SE-Phage-5 & - & + & +++ & +++ & +++ & +++ & +++ \\
\hline SE-Phage-6 & - & + & +++ & +++ & +++ & +++ & +++ \\
\hline SE-Phage-7 & - & - & +++ & +++ & +++ & +++ & +++ \\
\hline SE-Phage-8 & - & +++ & +++ & +++ & +++ & +++ & +++ \\
\hline SE-Phage-9 & - & - & +++ & +++ & +++ & +++ & +++ \\
\hline SE-Phage-10 & - & - & +++ & ++- & +++ & +++ & +++ \\
\hline SE-Phage-11 & - & + & +++ & - & +++ & +++ & +++ \\
\hline SE-Phage-12 & - & - & +++ & - & +++ & +++ & +++ \\
\hline SE-Phage-13 & - & +++ & +++ & +++ & +++ & +++ & +++ \\
\hline SE-Phage-14 & +++ & +++ & +++ & +++ & +++ & +++ & +++ \\
\hline SE-Phage-15 & - & - & +++ & +++ & +++ & +++ & - \\
\hline SE-Phage-16 & +++ & +++ & +++ & +++ & +++ & +++ & +++ \\
\hline SE-Phage-17 & - & +++ & +++ & +++ & +++ & +++ & - \\
\hline SE-Phage-18 & - & +++ & ++- & +++ & - & ++- & - \\
\hline SE-Phage-19 & - & +++ & +++ & +++ & +++ & +++ & +++ \\
\hline SE-Phage-20 & - & - & +++ & +++ & +++ & +++ & +++ \\
\hline SE-Phage-21 & - & - & - & - & +++ & ++- & - \\
\hline SE-Phage-22 & - & - & + & ++- & +++ & - & - \\
\hline SE-Phage-23 & - & - & ++- & +++ & ++- & + & - \\
\hline SE-Phage-24 & - & - & +++ & +++ & +++ & +++ & +++ \\
\hline SE-Phage-25 & - & - & +++ & - & +++ & +++ & +++ \\
\hline SE-Phage-26 & - & + & +++ & +++ & +++ & +++ & +++ \\
\hline SE-Phage-27 & - & - & +++ & +++ & +++ & +++ & +++ \\
\hline SE-Phage-28 & - & +- & +++ & - & +++ & +++ & +++ \\
\hline SE-Phage-29 & - & ++- & +++ & +++ & +++ & +++ & +++ \\
\hline SE-Phage-30 & +++ & + & +++ & +++ & +++ & +++ & +++ \\
\hline SE-Phage-31 & - & - & +++ & +++ & +++ & +++ & - \\
\hline SE-Phage-32 & - & - & +++ & ++- & +++ & +++ & +++ \\
\hline SE-Phage-33 & - & - & +++ & - & +++ & +++ & +++ \\
\hline
\end{tabular}


Table 4 (cont.)

Infective effect of Salmonella Enteritidis phages on Salmonella Enteritidis and Salmonella Typhimurium

\begin{tabular}{|c|c|c|c|c|c|c|c|c|}
\hline \multicolumn{3}{|c|}{ Salmonella Enteritidis } & \multicolumn{6}{|c|}{ Salmonella Typhimurium } \\
\hline 411 & 512 & 742 & SRII & $\operatorname{Tr} 90$ & Wild type & 625 & Aİ̈BB & 14028 \\
\hline+++ & - & +++ & + & - & +- & +- & +- & + \\
\hline+ & - & + & ++- & - & + & - & ++- & ++- \\
\hline+++ & - & +++ & - & - & +++ & + & ++- & +++ \\
\hline+++ & - & +++ & - & - & - & - & - & - \\
\hline+++ & - & +++ & + & - & + & + & + & + \\
\hline+++ & - & +++ & + & - & + & +- & +- & - \\
\hline+++ & - & +++ & + & - & +- & + & +- & +- \\
\hline+++ & - & +++ & + & - & - & + & +++ & - \\
\hline+++ & - & +++ & + & - & + & + & + & + \\
\hline+++ & - & +++ & + & - & + & + & - & + \\
\hline+++ & - & +++ & + & - & + & + & + & + \\
\hline+++ & - & +++ & + & - & + & + & + & + \\
\hline+++ & - & +++ & + & - & + & + & - & + \\
\hline+++ & - & +++ & + & - & +++ & + & ++- & +++ \\
\hline+++ & - & +++ & + & - & +++ & + & + & +++ \\
\hline+++ & - & +++ & ++- & - & + & + & + & + \\
\hline+++ & - & +++ & - & - & ++- & - & ++- & ++- \\
\hline+++ & - & +++ & + & - & +++ & + & +++ & +++ \\
\hline+++ & - & +++ & + & - & + & + & - & + \\
\hline+++ & - & +++ & - & - & - & - & - & - \\
\hline- & - & - & - & - & - & - & - & - \\
\hline- & - & - & - & - & - & - & - & - \\
\hline- & - & - & - & - & - & - & - & - \\
\hline+++ & - & +++ & + & - & + & + & + & + \\
\hline+++ & - & +++ & - & - & ++- & - & + & ++- \\
\hline+++ & - & +++ & - & - & +++ & + & ++- & +++ \\
\hline+++ & - & +++ & + & - & + & + & + & + \\
\hline+++ & - & +++ & - & - & - & + & - & - \\
\hline+++ & - & +++ & + & - & + & ++- & + & + \\
\hline+++ & - & +++ & - & - & + & ++- & - & ++- \\
\hline+++ & - & +++ & + & - & +++ & - & + & +++ \\
\hline+++ & - & +++ & - & - & - & - & - & - \\
\hline+++ & - & +++ & - & - & + & + & - & + \\
\hline
\end{tabular}


Table 4 (cont.)

\begin{tabular}{|c|c|c|c|c|c|c|c|}
\hline \multirow{2}{*}{ Phage } & \multicolumn{7}{|c|}{ Salmonella Enteritidis } \\
\hline & DMC3 & DMC8 & DMC22 & DMC31 & DMC94 & 13075 & 29 \\
\hline SE-Phage-34 & - & + & +++ & +++ & +++ & ++- & - \\
\hline SE-Phage-35 & - & +++ & +++ & +++ & +++ & +++ & - \\
\hline SE-Phage-36 & - & +++ & +++ & +++ & +++ & +++ & +++ \\
\hline SE-Phage-37 & +++ & ++- & +++ & +++ & +++ & +++ & ++- \\
\hline SE-Phage-38 & - & + & +++ & +++ & +++ & +++ & +++ \\
\hline SE-Phage-39 & +++ & ++- & +++ & +++ & +++ & +++ & +++ \\
\hline SE-Phage-40 & - & +++ & +++ & +++ & +++ & +++ & +++ \\
\hline SE-Phage-41 & - & +++ & +++ & +++ & +++ & +- & - \\
\hline SE-Phage-42 & - & - & +++ & +++ & +++ & +++ & - \\
\hline SE-Phage-43 & - & +- & +++ & +++ & +++ & +++ & +++ \\
\hline SE-Phage-44 & +++ & ++- & +++ & +++ & +++ & +++ & +++ \\
\hline SE-Phage-45 & - & - & - & +++ & ++ & + & - \\
\hline SE-Phage-46 & - & + & +++ & +++ & +++ & +++ & +++ \\
\hline SE-Phage-47 & - & +++ & +++ & +++ & +++ & +++ & +++ \\
\hline SE-Phage-48 & - & +- & +++ & +++ & +++ & +++ & +++ \\
\hline SE-Phage-49 & - & - & +++ & +++ & +++ & +++ & +++ \\
\hline SE-Phage-50 & - & + & +++ & +++ & +++ & +++ & +++ \\
\hline SE-Phage-51 & - & +++ & +++ & - & +++ & +++ & +++ \\
\hline SE-Phage-52 & - & - & +++ & +++ & +++ & +++ & +++ \\
\hline SE-Phage-53 & - & +++ & ++- & - & +- & - & - \\
\hline SE-Phage-54 & - & +++ & +++ & +++ & +++ & +++ & +++ \\
\hline SE-Phage-55 & - & - & +++ & +++ & +++ & +++ & +++ \\
\hline SE-Phage-56 & - & - & +++ & ++- & +++ & +++ & +++ \\
\hline
\end{tabular}

$+++, 10^{-2}, 10^{-4}$ and $10^{-6}$ diluted phage samples were inhibitor positive; ++-, $10^{-2}, 10^{-4}$ diluted phage samples were inhibitor positive but $10^{-6}$ dilution sample was inhibitor negative;,$+- 10^{-2}$ dilution sample was inhibitor positive, $10^{-4}$ and $10^{-6}$ dilution sample was inhibitor negative; $-, 10^{-2}, 10^{-4}$ and $10^{-6}$ diluted phage samples were inhibitor negative

Thompson, Anatum, Telaviv, Montavide, Kentucky and Carvalis, $75.8 \%$ of isolated ST-phages ( 25 out of 33 ) or $83.93 \%$ of SE-phages ( 47 out of 56 ) formed clear plaques and were capable to lyse at least six or four serovars, respectively. The rest of them formed turbid plaques, showing lysogeny or low possibility of killing each infected cell. Wide host range phages against $S$. Typhimurium or $S$. Enteritidis with clear plaques were predominantly isolated from sewage and wastewater from different sources. Beside Salmonella serovars, 15 out of 33 ST-phages (45.5\%) were also 
Table 4 (cont.)

\begin{tabular}{|c|c|c|c|c|c|c|c|c|}
\hline \multicolumn{3}{|c|}{ Salmonella Enteritidis } & \multicolumn{6}{|c|}{ Salmonella Typhimurium } \\
\hline 411 & 512 & 742 & SRII & $\operatorname{Tr} 90$ & Wild type & 625 & AİÜB & 14028 \\
\hline+++ & - & +++ & - & - & - & - & - & - \\
\hline+++ & - & +++ & - & - & ++- & + & ++- & ++- \\
\hline+++ & - & +++ & + & - & + & + & + & - \\
\hline+++ & - & +++ & ++- & - & + & + & + & + \\
\hline+++ & - & +++ & + & - & ++- & + & + & + \\
\hline+++ & - & +++ & ++- & - & + & + & + & + \\
\hline+++ & - & +++ & + & - & - & - & + & + \\
\hline+ & - & + & - & - & ++- & + & ++- & + \\
\hline+++ & - & +++ & + & - & +++ & - & + & +++ \\
\hline+++ & - & +++ & + & - & - & + & +- & - \\
\hline+++ & - & +++ & ++- & - & + & + & + & + \\
\hline- & - & - & - & - & - & - & - & - \\
\hline+++ & - & +++ & - & - & - & - & - & - \\
\hline+++ & - & +++ & + & - & + & + & + & + \\
\hline+++ & - & +++ & + & - & +++ & + & +++ & ++- \\
\hline+++ & - & +++ & + & - & +- & + & + & +- \\
\hline+++ & - & +++ & + & - & + & + & + & - \\
\hline+++ & - & +++ & - & - & - & + & - & - \\
\hline+++ & - & +++ & + & - & +- & + & + & +- \\
\hline- & - & - & - & - & - & - & - & - \\
\hline+ & - & +++ & - & - & ++- & + & ++- & +- \\
\hline+++ & - & +++ & - & - & - & - & - & - \\
\hline+++ & - & +++ & + & - & + & + & - & + \\
\hline
\end{tabular}

infective against $E$. coli strains including E. coli O157:H7. ST-Phage-6, 3 and 10 had the broadest host range, with lytic ability against 18 to 17 different bacterial strains (lysed $50-47 \%$ of the strains tested), 8-9 of them from $S$. Typhimurium, 7-8 from other $S$. enterica serovars and 1-2 from E. coli strains. These were followed by ST-Phage-4, 8 and 9, being infective against 16-15 different bacterial strains. Two $S$. Typhimurium strains (TH3923, SL134) were lysed by most of the isolated phages, possibly due to the lack of prophage in these isolates. ST-Phage-5, 9, 24, 25, 27, 28 and 30 were infective against 3 different strains of E. coli $0157: \mathrm{H} 7$ besides Salmonella serovars. 
As seen in Tables 3 and 4, 80.36\% (45 out of 56) and 12.5\% (7 out of 56) of SE-phages were effective against $S$. Typhimurium and other Salmonella enterica serovars, respectively, besides their host cell serovars. Only 7.14\% of SE-phages (SE-Phage-2, 10, 22, 32) were infective against E. coli strains. SE-Phage-3 had the broadest host range, having lytic activity against 15 different bacterial strains (lysed $51.7 \%$ of the strains tested), $9 \mathrm{~S}$. Enteritidis, 5 other $S$. enterica serovars and 1 E. coli (Tables 3 and 4). This was followed by SE-Phage-1, 14, 16, 37, 44, and 48, being infective against 14 different bacterial strains. Eight SE-Phages were infective most $S$. Enteritidis serovars tested except $S$. Enteritidis METU S1-512 (Table 4). It was observed that $S$. Enteritidis METU S1-512 was not affected by any SE-phages isolated (Table 4). None of the ST- and SE-phages were found to be infective against, Y. enterocolitica, C. freundii, E. aerogenes and Gram-positive bacteria tested. These results show that ST-phages have wider host ranges than SE-phages. Neither $S$. Typhimurium nor Enteritidis phages were infective against Listeria monocytogenes, Staphylococcus aureus, Bacillus cereus, Yersinia enterocolitica, Citrobacter freundii, Enterobacter aerogenes and Enterococcus faecalis.

\section{DISCUSSION}

In the present study, lytic and broad spectrum $S$. Typhimurium or $S$. Enteritidis specific bacteriophages were isolated from natural sources, purified and developed for a phage collection to biocontrol their foodborne pathogenic host cells. Phages are the most common microorganisms in the world because they are an important part of both the intestinal and environmental ecosystems [22]. The existence of bacteriophages is closely interrelated with their natural hosts. Salmonella enterica serovars are natural inhabitants of the gastrointestinal tract of all animals and abundant in animal feces. Because Salmonella serovars can be transmitted easily to various waste effluents, sewages and wastewaters are certainly the best sources for anti-Salmonella phage isolation [14]. The richest sources in terms of phages that were active against $S$. Typhimurium or $S$. Enteritidis strains tested were sewage, slaughterhouse wastewaters, food processing wastewaters, streams and fisheries wastewater. Surprisingly, raw foods (milk, meat, fruits and vegetables) and troughs were found to be quite poor in terms of antiSalmonella phage. $S$. Typhimurium bacteriophages were detected in only 2 meat samples, which were lysogenic. Three anti- $S$. Enteritidis phages were found on chicken meat samples, but their host spectrum was very narrow, being effective on three different $S$. Enteritidis strains. The phage infecting $S$. Typhimurium MA1LT2/pNK972, MA53 T-POP and SL134 or $S$. Enteritidis METU S1512 was not detected. These results show how important the number of strains or serovars to be used in the bacteriophage screening is. Similar to our results, several studies have reported that sewages or wastewaters are very rich sources of phages infecting Salmonella serovars [2, $8,21,22,25]$. Another rich source is reported to be animal feces $[2,4,13,23]$. In the present study, it was found that the richest resources in terms of $S$. Typhimurium or Enteritidis phages are sewage, food processing and animal wastewater. 
For isolation of bacteriophage, enrichment method is better than direct method especially if the phage number is low. Similar results were reported by Akhtar et al. [2] and McLaughlin et al. [18].

Host specificity being restricted to particular strains within a single bacterial species is a common property of bacteriophages, but it is a limiting factor for food industry to control foodborne pathogens or spoilage bacteria and therapeutic treatment of bacterial infections in medicine [5]. In this study, the isolated anti-S. Typhimurium or $S$. Enteritidis phages showed wide host ranges and were able to lyse 3 to 18 or 2 to 15 of 36 strains tested, respectively. Moreover, even though ST-phages were isolated using $S$. Typhimurium strains, some of them were also able to infect and lyse other $S$. enterica serovars including $S$. Enterititis and $E$. coli $\mathrm{O} 157: \mathrm{H} 7$ which are significant pathogenic bacteria in the food industry. The isolated most SE-phages were also effective against $S$. Typhimurium besides their host cell serovars.

Similar to our results, some known Salmonella bacteriophages have wide host ranges such as SSP5 and SSP6 bacteriophages [15], PSPu-95 and PSPu-4-116 [3] and Felix 01 [16]. O'Flynn et al. [20] also reported that phages st104a and st104b had a broad host range within the $S$. enterica species including Typhimurium, Enteritidis, Newport and Derby.

Bao et al. [4] stated that anti-S. Enteritidis phages PA13076 and PC2184 isolated from chicken sewages both had wide host ranges and phage PA13076 had a lytic effect on 222 of the 311 epidemic Salmonella isolates (71.4\%), whereas PC2184 showed a lytic effect on 298 isolates (95.8\%).

It has also been demonstrated by a number researchers that phages isolated from sewage or waste water have wide infective spectra and do not have high serovar specificity [2, 8]. Santos et al. [24] informed that PVP-SE1, a lytic $S$. Enteritidis phage, isolated from wastewater plant infects a wide variety of Salmonella serotypes isolated from different sources (food, environmental and clinical) and E. coli. On the other hand, Carey-Smith et al. [8] reported that FGCSSal phage only infect six of eight Salmonella hosts but did not infect the E. coli tested.

In conclusion, the wide host ranges of the isolated anti-S. Typhimurium and Enteritidis phages can allow the successful application to prevent the contamination of $S$. Typhimurium and $S$. Enteritidis in food. Furthermore, they can be used as a possible alternative to chemical antimicrobials in food industry and to antibiotics for the reduction of $S$. Typhimurium or $S$. Enteritidis infections in animal breeding, especially poultry breeding. Further research will be conducted to identify these antiSalmonella phages using morphological, physical, biochemical and molecular techniques, and to determine their suitability for use in the food industry as biocontrol agents and therapy agents in animal infection.

\section{ACKNOWLEDGEMENTS}

This research was supported by a grant from the Scientific and Technological Research council of Turkey (TÜBITAK) with project No: 2130035. 


\section{REFERENCES}

1. Adams, M. H. (1959) Bacteriophages. New York: Interscience Publishers, Inc.

2. Akhtar, M., Viazis, S., Diez-Gonzalez, F. (2014) Isolation, identification and characterization of lytic, wide host range bacteriophages from waste effluents against Salmonella enterica serovars. Food Control 38, 67-74.

3. Bao, H., Zhang, H., Wang, R. (2011) Isolation and characterization of bacteriophages of Salmonella enterica serovar Pullorum. Poultry Sci. 90, 2370-2377.

4. Bao, H., Zhang, P., Zhang, H., Zhou, Y., Zhang, L., Wang, R. (2015) Bio-control of Salmonella Enteritidis in foods using bacteriophages. Viruses 7, 4836-4853.

5. Bielke, L. R., Higgins, S. E., Donoghue, A. M., Donoghue, D. J., Hargis, B. M., Tellez, G. (2007) Use of wide-host-range bacteriophages to reduce Salmonella on poultry products. Int. J. Poultry Sci. $6,754-757$.

6. Borie, C., Albala, I., Sánchez, P., Sánchez, M. L., Ramírez, S., Navarro, C. (2008) Bacteriophage treatment reduces Salmonella colonization of infected chickens. Avian. Dis. 52, 64-67. 10.1637/8091082007-Reg.

7. Callaway, T. R., Edrington, T. S., Brabban, A., Kutter, E., Karriker, L., Stahl, C., Wagstrom, E., Anderson, R. C., Genovese, K., McReynolds, J. (2010) Occurrence of Salmonella-specific bacteriophages in Swine feces collected from commercial farms. Foodborne Pathog. Dis. 7, 851-856.

8. Carey-Smith, G. V., Billington, C., Cornelius, A. J., Hudson, A., Heinemann, J. A. (2006) Isolation and characterization of bacteriophages infecting Salmonella spp. FEMS Microbiol. Lett. 258, $182-186$.

9. Chanishvili, N., Chanishvili, T., Tediashvili, M., Barrow, P. A. (2001) Phages and their application against drug-resistant bacteria. J. Chemical. Technol. Biotechnol. 76, 689-699.

10. Connerton, P. L., Connerton, I. F. (2005) Microbial treatments to reduce pathogens in poultry meat In: Mead, G. (ed.) Food Safety Control in the Poultry Industry, Woodhead Publishing Ltd., Cambridge, UK, pp. 414-427.

11. Helms, M., Ethelberg, S., Mølbak, K. (2005) International Salmonella Typhimurium DT104 infections, 1992-2001. Emerg. Infec. Dis. 11, 859-867.

12. Huff, W. E., Huff, G. R., Rath, N. C., Balog, J. M., Donoghue, A. M. (2005) Alternatives to antibiotics: Utilization of bacteriophage to treat colibacillosis and prevent foodborne pathogens. Poultry Sci. $84,655-659$.

13. Hungaro, H. M., Mendonça, R. C. S., Gouvêa, D. M., Vanetti, M. C. D., de Oliveira Pinto, C. L. (2013) Use of bacteriophages to reduce Salmonella in chicken skin in comparison with chemical agents. Food Res. Int. 52, 75-81.

14. Guttman, B., Raya, R., Kutter, E. (2005) Basic phage biology. Sulakvelidze, E., Boca, A. (eds) Bacteriophages, Biology and Applications. Raton: CRC Press, pp. 29-66.

15. Kocharunchitt, C., Ross, T., McNeil, D. L. (2009) Use of bacteriophages as biocontrol agents to control Salmonella associated with seed sprouts. Int. J. Food Microbiol. 128, 453-459

16. Kostrzynska, M., Campos, M. C., Griffiths, M., Lepp, D. (2002) Biocontrol of Salmonella enterica serovar Typhimurium DT104 on poultry products using bacteriophages. In Proc. Agric. Agric.-Food Canada Food Network Meet., Lacombe, Alberta, Canada. p. 35.

17. Mahony, J., Mauliffe, O., Ross, R. P., van Sinderen, D. (2011) Bacteriophages as biocontrol agents of food pathogens. Curr. Opinion. Biotechnol. 22, 157-163.

18. McLaughlin, M. R., Balaa, M. F., Sims, J., King, R. (2006) Isolation of Salmonella bacteriophages from swine effluent lagoons. J. Environ. Qual. 35, 522-528.

19. Monk, A., Rees, C., Barrow, P., Hagens, S., Harper, D. (2010) Bacteriophage applications: where are we now? Lett. Appl. Microbiol. 51, 363-369.

20. O'Flynn, G., Coffey, A., Fitzgerald, G. F., Ross, R. P. (2006) The newly isolated lytic bacteriophages st104a and st104b are highly virulent against Salmonella enterica. J. Appl. Microbiol. 101, 251-259. 
21. O’Flynn, G., Ross, R. P., Fitzgerald, G. F., Coffey, A. (2004) Evaluation of a cocktail of three bacteriophages for biocontrol of Escherichia coli O157:H7. Appl. Environ. Microbiol. 70, 3417-3424.

22. Oliveira, A., Sillankorva, S., Henriques, A., Quinta, R., Sereno, R., Azeredo J. (2009) Isolation and characterization of bacteriophages for avian pathogenic E. coli strains. J. Appl. Microbiol. 106, 1919-1927.

23. Raya, R. R., Varey, P., Oot, R. A., Dyen, M. R., Callaway, T. R., Edrington, T. S., Kutter, E. M., Brabban, A. D. (2006) Isolation and characterization of a new T-even bacteriophage, CEV1, and determination of its potential to reduce Escherichia coli O157:H7 levels in sheep. Appl. Environ. Microbiol. 72, 6405-6410.

24. Santos, S. B., Fernandes, E., Carvalho, C. M., Sillankorva, S., Krylov, V. N., Pleteneva, E. A., Shaburova, O. V., Nicolau, A., Ferreira, E. C., Azeredo, J. (2010) Selection and characterization of a multivalent Salmonella phage and its production in a nonpathogenic Escherichia coli strain. Appl. Environ. Microbiol. 76, 7338-7342.

25. Sillankorva, S., Pleteneva, E., Shaburova, O., Santos, S., Carvalho, C., Azeredo, J., Krylov, V. (2010) Salmonella Enteritidis bacteriophage candidates for phage therapy of poultry. J. Appl. Microbiol. 108, 1175-1186.

26. Stenholm, A. R., Dalsgaard, I., Middelboe, M. (2008) Isolation and characterization of bacteriophages infecting the fish pathogen Flavobacterium psychrophilum. Appl. Environ. Microbiol. 74, 4070-4078.

27. Tsonos, J., Vandenheuvel, D., Briers, Y., De Greve, H., Hernalsteens, J. P., Lavigne, R. (2013) Hurdles in bacteriophage therapy: deconstructing the parameters. Vet. Microbiol. 171, 460-469.

28. Velge, P., Cloeckaert, A., Barrow, P. (2005) Emergence of Salmonella epidemics: the problems related to Salmonella enterica serotype Enteritidis and multiple antibiotic resistance in other major serotypes. Vet. Res. 36, 267-288. 
